\title{
IMPLEMENTATION OF NUTRITION SCREENING FOR OLDER ADULTS IN GENERAL PRACTICE: PATIENT PERSPECTIVES INDICATE ACCEPTABILITY
}

\author{
A.H. Hamirudin ${ }^{1}$, K. Charlton ${ }^{1}$, K. Walton ${ }^{1}$, A. Bonney 1 , G. Albert ${ }^{2}$, A. Hodgkins ${ }^{3}$, A. Ghosh ${ }^{4}$, \\ J. Potter ${ }^{5}$, M. Milosavljevic ${ }^{6}$, A. Dalley
}

\begin{abstract}
Background: Older patients' views regarding undergoing nutrition screening within General Practice settings have not been evaluated to date. Objectives: To identify perceptions of older patients related to their experiences of having a nutrition screening process performed using the Mini Nutritional Assessment Short Form (MNA-SF®), accompanied by a clinical care pathway. Methods: Patients aged $\geq 75$ years were invited to attend repeat screening between 6 months and one year following a first screening $(n=143)$. Patients who were identified to be malnourished or at risk at baseline $(n=44)$ were invited to participate in an individual interview to identify their perceptions of the MNA-SF® and the applicability of a nutrition resource kit that had been provided to them. Results: Nutritional status improved in the group identified to be malnourished/at risk at baseline ( $\mathrm{p}=0.01$ ). Interviews indicated that the MNA-SF® process was well-received but that patients did not perceive themselves as being in need of nutrition support. Conclusion: This study demonstrates that introduction of routine nutrition screening of older patients attending General Practice can feasibly be implemented using the MNA-SF® and is acceptable to patients. It is recommended that this model of care be adopted in order to improve early identification of nutritional risk and facilitate referral to appropriate services.
\end{abstract}

Key words: Malnutrition, older adults, nutrition screening, general practice, outcomes.

\section{Introduction}

Malnutrition that develops in older adults living in community settings contributes to both increased hospital and residential care admissions (1). Malnourished older adults have a slower recovery, longer hospital stays, reduced quality of life and more frequent visits to their general practitioners (GPs) (2). Timely identification and management of malnutrition in this age group through routine nutrition screening can prevent further deterioration in nutritional status (3-5). In Australia and other countries, clinical guidelines recommend performing nutrition screening in older adults across all settings, including primary health care

1. School of Medicine, Faculty of Science, Medicine and Health, University of Wollongong, NSW , Australia and Illawarra and Southern Practice Research Network; 2. Shellharbour Medical Centre, NSW, Australia and Illawarra and Southern Practice Research Network; 3. Junction Street Family Practice and Illawarra and Southern Practice Research Network; 4. CO-ORDINARE South Eastern NSW PHN, NSW, Australia NSW; 5. Division of Aged Care, Rehabilitation and Palliative Care, Illawarra Shoalhaven Local Health District, NSW, Australia; 6. Research Directorate, Illawarra Shoalhaven Local Health District, NSW, Australia; 7. General Practitioner

Corresponding Author: Karen Charlton, School of Medicine, Faculty of Science, Medicine and Health, University of Wollongong, Wollongong NSW 2522, Tel: +61 24221 4754, Fax: +61 24221 3486, Email: karenc@uow.edu.au

Received September 1, 2015

Accepted for publication September 2, 2015
(6-9).

In the UK, nutrition screening was reportedly poorly performed in general practice (9) until the introduction of a validated nutrition screening tool (10). A lack of knowledge related to the process of nutrition screening and nutrition care pathways as well as a lack of appropriate patient education resources have previously been outlined as barriers to performing nutrition screening by Australian general practitioners and practice nurses (11). Opportunities identified by practitioners include the incorporation of a nutrition screening tool within current practice, via the existing Medicare-funded Health Assessment for older persons aged 75 years and older (75+ HA) (11), as has been advocated by others (12, 13)

Implementation of nutrition screening in general practice has been demonstrated to be feasible, provided that appropriate training and resources are provided by dietitians, within a multidisciplinary approach (14 ).This involves the use of a validated screening tool (2) that is accompanied by initiation of clear clinical pathways associated with the identified screening classification (3). Participatory action research with Australian general practitioners has identified that an electronic format of 
the Mini Nutrition Assessment Short Form (MNA-SF®) is the preferred version of the screening tool since it can be linked to patient medical records within existing desktop clinical software packages (14).

To date, the views of older patients themselves regarding their experiences of the screening process in general practice has not been explored. This mixed methods study was performed to assess older patients' perceptions related to the MNA-SF®, screening process and the acceptability and usefulness of nutrition resources that had been provided. Secondary aims were to assess change in nutritional status and within 6 months to 1 year following the initial nutrition screening and to identify factors that were associated with the likelihood that patients would be malnourished or 'at risk' at follow up.

\section{Methods}

Three General Practices that are members of the Illawarra and Southern Practice Research Network (ISPRN) participated in this study and were from a regional, rural and metropolitan area within the Illawarra and Shoalhaven Medicare Local catchment area of New South Wales, Australia.

For a period of three months following the completion of a training session on how to perform nutrition screening using MNA-SF®, 143 older patients aged $\geq 75$ years were screened by practice staff within the $75+\mathrm{HA}$ and opportunistically for those attending the practice for consultations. Patients who were identified to be 'at risk of malnutrition' or 'malnourished' were provided with a resource kit and other interventions, as outlined in the MNA-SF® nutrition intervention pathway guide (15). The care pathway recommends to treat 'malnourished' and 'at risk patients' by referring them to an accredited practising dietitian; whilst annual nutrition screening is recommended to well-nourished community living older adults. Nutrition resource kits that had been specifically developed for use in the geographical region of each general practice were provided to practice staff for the purpose of patient education. The kits included a leaflet about high energy and high protein foods, an 'Eating Well' booklet (16), a relevant local council directory of nutrition-related services and available support services for older persons in their catchment area.

All 143 of the patients aged 75+ years who had undergone screening were invited for a nutrition screening follow up visit at their General Practice within 6 months to one year of the first screening (Figure 1). Each patient received a personal letter outlining the results of their initial nutrition screening [Scores between 12-14 indicate 'well- nourished', 8-11 'at risk' and '0-7' as 'malnourished' (17)] and were invited to attend for a follow up screening interview conducted by practice nurses at the participating General Practices. The MNA$\mathrm{SF}{ }^{\circledR}$ was repeated using the electronic format, the results of which were incorporated into patients' electronic medical records.

Patients who were categorised as 'malnourished' or 'at risk of malnutrition' $(\mathrm{n}=44)$ at their initial screen were invited to participate in an individual in-depth interview to assess patients' perceptions of the MNA-SF ${ }^{\circledR}$ and nutrition screening process, and to obtain feedback on the nutrition resource kit that had been provided. Interviews were conducted using open-ended questions (18) by a single dietitian researcher on the same day as the nutrition screening follow up at each general practice. Interviews took approximately 30 minutes and were audio recorded, transcribed verbatim, coded into topics and analysed thematically using qualitative analysis software (QSR NVivo version 10).

Referral patterns to services and information on clinical outcomes, such as hospitalisation since first screening were extracted from individual patient's electronic medical records by practice nurses in order to assess associations with nutritional status at follow up. Predictors of being 'at risk' and 'malnourished' at follow up and other process outcomes were analysed using binary logistic regression modelling. Wilcoxon Signed Ranks Tests were performed to analyse differences in MNA-SF® score as data was not normally distributed. Statistical analyses were conducted using IBM SPSS version 21. Statistical significance was set at $p<0.05$.

Ethics approval for the study was granted by University of Wollongong Human Research Ethics Committee (HE12/381) and written consent was given by all participants.

\section{Results}

Seventy-two patients of the initial $143(50.3 \%)$ participated in follow up screening (Clinic $1: \mathrm{n}=38 / 74$ $(51.4 \%)$, Clinic 2: $\mathrm{n}=15 / 27(55.5 \%)$ and Clinic 3: $\mathrm{n}=$ $19 / 42(45.2 \%))$. The MNA-SF® scores for patients who completed follow-up screening are shown in Table 1 , as categories of nutritional risk. At follow-up, 20 participants $(27.8 \%)$ were identified as 'at risk'; while one patient $(1.4 \%)$ was malnourished and 51 patients $(70.8 \%)$ were well-nourished.

Seventeen of the forty-four patients $(38.6 \%)$ who had been identified as 'malnourished' or 'at risk' in the initial screening participated in an in-depth interview (Clinic 1: $n=6$; Clinic 2: $n=9$; and Clinic 3: $n=2$ ); whilst three patients refused to be interviewed. Sixteen of the interviews were audio recorded, transcribed verbatim, coded into topics and analysed thematically. Data saturation was achieved by the fifteenth interview, although all 16 interviews were analysed. Gender (Male: M, Female: F), participants' code and, MNA-SF® scores at initial screening (1st) and follow-up (2nd) of patients are indicated with the exemplar quotes for each theme. 


\section{Patients' perspectives on MNA-SF® and the nutrition screening process}

Three emergent themes were identified.

\section{Theme 1: Well-received}

Patients were willing to answer the items in the MNA$\mathrm{SF}{ }^{\circledR}$ instrument, because they perceived the questions to be non-confrontational and not to be of a sensitive nature, and were therefore perceived to have no adverse repercussions.

'That doesn't worry me one iota' (F7), (1st screen MNA-SF® score $=10,2$ nd screen score $=12$ )

The MNA-SF® was perceived to be a simple tool and was thought to be beneficial for improving the nutritional care of older patients.

'Well it's quite simple. When you get to my age, you want things simple don't you?' (M1), (1st 11, 2nd 12)

'It may be beneficial to all old people I suppose to be quite honest and if things are required after that well it'd most probably be a good thing you know' (M2),(1st 11, 2nd 14)

\section{Theme 2: Lack of concern about nutrition screening}

The results of the nutrition screening process did not appear to be considered a priority for many participants. Categorisation of being 'at risk' or 'malnourished' had no influence on patients' behaviour relating to their dietary habits. There was a perception that a decline in nutritional status was considered to be a normal part of the ageing process

'Well they can't do much. It's me getting old, tired and worried and well, you know.'(F2), (1st 7, 2nd 10)

Prioritisation of family and social issues downplayed the importance of the nutritional screening results.

'Well because of the worry I have with my son and his children, I didn't really take an awful lot of notice of it I'm afraid. I'm sorry, I should have but I didn't.' (F4), (1st 6, 2nd 7)

Other concerns that carried higher priority included current medical conditions.

'I don't have trouble with any of that. The trouble is if I eat too much it sits here, you know, I can't digest it so I just do what I think is right, you know, what I'm comfortable with and we have cereal for breakfast which is Plus.' (F3), (1st 9, 2nd 13)

Figure 1

Research protocol

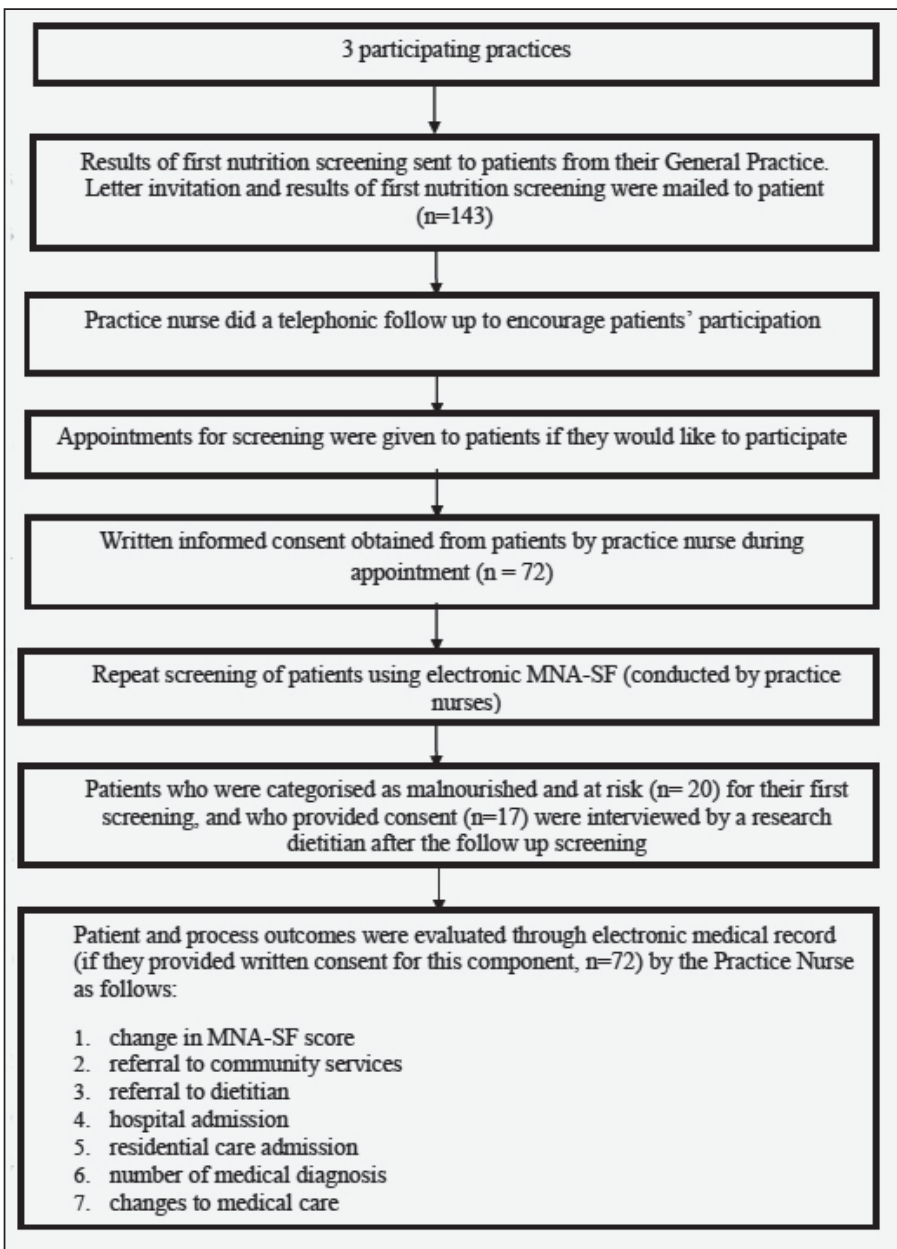

\section{Theme 3: Conscious about MNA-SF® items}

Some participants expressed disappointment with their MNA-SF® score, as they believed that they were eating well.

'Well I couldn't understand that. When I eat properly - I feel I eat properly - I couldn't understand why...then it showed that I was malnourished.' (F5), (1st 8, 2nd 14).

Table 1

Number of patients who completed both initial and follow up MNA-SF® screening

\begin{tabular}{lcccccc}
\hline Categories & \multicolumn{2}{c}{ Clinic 1 (regional) (n= 38) } & \multicolumn{2}{c}{ Clinic 2 (metropolitan) (n= 15) } & \multicolumn{2}{c}{ Clinic 3 (rural) (n= 19) } \\
& Initial screening & Follow-up & Initial screening & Follow-up & Initial screening & Follow-up \\
\hline Malnourished & 0 & 0 & 2 & 1 & 0 & 0 \\
At risk & 7 & 15 & 7 & 5 & 4 & 0 \\
Well-nourished & 31 & 23 & 6 & 9 & 15 & 19 \\
Total & 38 & 38 & 15 & 15 & 19 \\
\hline
\end{tabular}


Table 2

Mean (SD) MNA-SF® score based on groups

\begin{tabular}{|c|c|c|c|}
\hline MNA-SF Score & $\begin{array}{l}\text { Initial screening } \\
\qquad \text { Score }\end{array}$ & $\begin{array}{l}\text { Follow-up } \\
\text { Score }\end{array}$ & P value \\
\hline $\begin{array}{l}\text { Malnourished and at risk of malnutrition }(n=20) \text { (MNA-SF score } \\
<11)\end{array}$ & $9.9 \pm 1.5($ Range $=6-11)$ & $11.4 \pm 2.1($ Range $=7-14)$ & $0.01^{*}$ \\
\hline Well-nourished $(\mathrm{n}=52)(\mathrm{MNA}-\mathrm{SF}=12-14)$ & $13.3 \pm 0.9($ Range $=12-14$ & $12.8 \pm 1.5($ Range $=9-14)$ & 0.071 \\
\hline
\end{tabular}

\section{Patient perceptions of the resource kit}

Five emergent themes were identified. Nine participants stated that they had not received the resource kit.

\section{Theme 1: Support not required}

Fourteen participants perceived that nutrition supports were not required. Participants felt that they were eating the right types of food and did not require any additional information about services to further improve their nutritional status. This reflected a desire for independence.

'I don't need it. No, we look after ourselves as far as cooking and eating is concerned. I think common sense has got a lot to do with it. I am cutting down a little on the amount of red meat we eat but I decided that by myself. Well, we don't need so much red meat. We eat a lot of chicken' (F6), (1st 10, 2nd 10)

In addition, community-based services such as Meals on Wheels (MOW) were not considered to be a choice for the time being, as patients felt that they could still prepare their own food and expressed a desire to retain their independence.

'I have tasted the food, yes, but it's quite nice but... Not at the moment. Not at the moment while I can do things myself. (M1), (1st 11, 2nd 12)

Some patients expressed a dislike of oral nutrition supplementation (ONS), because of bad experiences of their peers.

'I don't like it (ONS). I'd rather be dead' (F10), (1st 11, 2nd 9)

\section{Theme 2: Existing clients of services}

Clients of MOW reported that they found the service as helpful, particularly as a standby on days when extra assistance was required, whilst five of them had seen a dietitian.

'We've got some in the fridge at the moment but I say to them for a sort of emergency when I can't be bothered cooking. If I get too tired or something happens to prevent me doing what I planned to do and then we do that, we use those; they're a stand-by really.'(M6), (1st 11, 2nd 11)

'Yes, well I've got to see her (dietitian) again. I've been seeing her about every three months in the last possibly 18 months, two years I suppose.' (M2), (1st 11, 2nd 14)

Few patients reported receiving home-help services for assistance with household chores and activities of daily living; and transitional care service when discharged home from hospital. One patient was a carer to an unwell spouse.

'My husband's fully assessed (diagnosed with cancer) so we can have whatever we need doing and I do have a home help comes in once a fortnight for kitchen, bathroom, toilet.' (F9), (1st 11, 2nd 11)

\section{Theme 3: Disregard of information provided}

Information that was provided was disregarded.

'I didn't follow it. No, I didn't actually - she (practice nurse) told me what cereal to take in the morning but I tried it - one plateful but I couldn't eat it. (F4), (1st 6, 2nd 7)

'Well, I haven't sat down to read them because I don't have enough vision for reading.' (F3), (1st 9, 2nd 13)

Patients also felt comfortable in continuing to do things their own ways, rather than taking the advice of a dietitian.

'Well yes, when X came out of hospital after the cancer operation we saw a dietitian about two or three times. As I said, the advice she gave us, well-meaning, but I didn't consider it all that helpful.' (M6), (1st 11, 2nd 11)

'I've found that any advice from the dietitians isn't all that helpful. It just confuses things really - I feel anyway.' (F10), (1st 1, 2nd 9)

\section{Theme 4: Informative}

Patients who had received the resource kit mentioned that it was informative, comprehensive and useful.

11)

'It's quite informative, very good.' (M5), (1st 11,2nd

'I thought they were very good. Yes, very informative.' (F5), (1st 8, 2nd 14)

Carer to patients perceived the kit as helpful to improving patients' nutritional status.

'It would probably be more helpful to us. Yes, to read 
Table 3

Logistic regression predicting likelihood of being 'malnourished' and 'at risk'

\begin{tabular}{|c|c|c|c|c|}
\hline \multirow[t]{2}{*}{ Process outcomes ( $n=$ number of occasion) } & \multirow[t]{2}{*}{ Odds ratio } & \multicolumn{2}{|c|}{$95 \%$ C.I. for Odds ratio } & \multirow[t]{2}{*}{ P value } \\
\hline & & Lower & Upper & \\
\hline Community Service Referral $(n=15)$ & 0.19 & 0.04 & 0.86 & $0.03^{*}$ \\
\hline Hospital Admission $(\mathrm{n}=17)$ & 1.02 & 0.20 & 5.26 & 0.98 \\
\hline Nursing home admission $(\mathrm{n}=2)$ & 0.00 & 0.00 & & 1.00 \\
\hline Medical care changes $(n=22)$ & 0.61 & 0.15 & 2.53 & 0.50 \\
\hline
\end{tabular}

${ }^{*} \mathrm{p}<0.05$

it to make sure that we can follow as many of these guidelines, suggestions that are outlined in the booklets.' (Son of F8), (1st 11, 2nd 9)

\section{Theme 5: Welcoming of new information}

Patients who didn't receive the resource kit were open to obtaining new information on nutrition and community services, when the kit was shown to them during interview. They felt that the information may help them with their current needs.

'If I get that book well that will help...things in there that don't occur to me.' (F4), (1st 6, 2nd 7)

'I'd like to have that one.' (M4), (1st 9, 2nd 12)

\section{Nutritional outcomes after screening}

A statistically significant improvement was found for change in mean MNA-SF® score for those patients categorised as malnourished and at risk at initial screening $(p=0.01)$ (Table 2$)$. Although an improvement in the mean total MNA-SF® score was detected, the mean score remained in the at-risk category (score $\geq$ 8-11), but closer to the well-nourished category. One malnourished patient at initial screening was identified as at-risk at follow up, whilst another patient remained malnourished. 11 patients who were at-risk initially had improved to the well-nourished category at follow up; nevertheless another 7 patients were still in the at-risk category.

No significant changes were detected in mean MNASF® score for the group who were well-nourished at initial screening $(\mathrm{p}=0.07)$. However, 12 of this group had a score that was in the at-risk category at follow up, indicating nutritional decline.

All 72 patients provided informed consent for access to their clinical records. Binary logistic regression analysis was performed to assess the impact of factors on the likelihood that patients would be identified as malnourished or 'at risk' at follow up. The model containing all predictors was statistically significant, $\chi 2$ $(6, \mathrm{~N}=72)=13.0, \mathrm{p}=0.043$. Only referral to community services significantly predicted the likelihood of being 'at risk' or 'malnourished' at the follow up with an odds ratio of $0.19(\mathrm{p}=0.03)$ (Table 3$)$.

\section{Discussion}

This study demonstrated that nutrition screening, followed up with appropriate referral and/or nutrition intervention within one year was associated with improved nutritional status of older patients' who were at risk of malnutrition, or malnourished at an initial screen. A mixed-methods approach provides a greater understanding in health care services research than using a single method (19). This is necessary to improve the nutrition service delivery model particularly in general practice as nutrition screening in older adults is not currently routinely performed (11).

This study demonstrated that the MNA-SF® was well-received among older patients attending Australian general practices and that the MNA-SF® questions were viewed as non-threatening by those who took part. However, most participants had little concern about improving their nutritional status. Low self-perceived health status has been reported to be associated with malnutrition risk (20) and this was apparent in some participants who felt that nothing could be done to improve their nutritional status due to their underlying current health conditions. Our study has identified a low level of awareness about the adverse consequences of being malnourished in community dwelling older adults, despite malnutrition being associated with a higher risk of hospital admission and longer hospital stay (21), as well as with a more than threefold mortality rate post hospital discharge, as compared to age-matched wellnourished older adults (22).

Interviews with malnourished and at risk patients suggested they did not perceive a need for additional information and nutrition support as they felt that they 
already knew how to eat well and look after themselves. An Australian study reported that general practitioners initiated dietetic referral for patients who were willing to modify their eating behaviour and could afford to pay additional charges (23) for those who were not entitled to a General Practice Management Plan (GPMP) or Team Care Arrangement (TCA) (24). This may be the case for this study as not all patients received dietetic intervention. Although some patients had been seen by dietitians, making dietary changes is ultimately an individual decision. A previous Australian study indicated that $56 \%$ of at risk community dwelling older adults refused a dietetics referral (25), further emphasising the difficulty in engaging this group, and a need for other strategies such as a multidisciplinary team approach (26). As suggested by others as being an effective strategy to encourage dietary behaviour change (27), we specifically developed a resource kit for each local area in which the participating general practices were situated. This was perceived as being informative in this study.

It is noteworthy that this lack of desire to receive nutritional intervention existed, given that over a quarter of patients $(28 \%)$ were identified to be at risk of malnutrition, a figure that is consistent with our previous work (14), and that of others $(28,29)$. Our study demonstrated that even patients who were identified as well-nourished at their initial screening were susceptible to experience a decline in their nutritional status over the period of up to a year, thus supporting the recommendation for routine annual nutrition screening in older adults in the community (15).

Of interest is the finding that referral to community services was associated with a greater likelihood of being 'malnourished' or 'at risk' at follow up. This is clinically plausible as patients are generally referred to services such as home help and respite when selfcare becomes problematic. Receiving home help (30) and a declining ability to perform Activities of Daily Living (31) are strong predictors of being malnourished, while older adults in receipt of home care services have a higher prevalence of malnutrition compared to their counterparts not in receipt of such services $(20,21,32)$. A hospital admission has been reported to be associated with a 1.8 fold increased risk of being undernourished (31), but was not identified as being significant in our study, possibly due to the short follow up period.

There are a number of limitations to our study. Only three general practices were recruited to administer and test the model of nutritional care. The 50\% drop out rate contributed to a small sample size which further limits generalisability of the findings. However, the qualitative approach has provided valuable insights into perceptions of older adults with regard to uptake of nutrition screening and its perceived benefits. Further investigation into older adults' nutritional needs and their motivation to change is warranted; as there was a widespread lack of awareness and concern regarding their compromised nutritional status.

\section{Conclusion}

This study has demonstrated that the MNA-SF® can be widely used in general practice and that nutrition screening of older adults, accompanied with nutrition intervention and provision of relevant resources is associated with improved nutritional status in this age group. Annual nutrition screening is strongly recommended for older adults to ensure optimum nutritional status. Practice nurses can play a leading role in performing nutrition screening in the general practice setting as has been modelled in this study with support from GPs and dietitians. Patients were accepting of the MNA-SF®, however further research is needed to test this model of care and the implementation of strategies to improve uptake of appropriate nutrition support by older patients.

Acknowledgement: This study was funded by 2011 Illawarra Health and Medical Research Institute (IHMRI) Clinical grant. We would like to thank general practice staff, participants and local councils for contributions to this study.

Conflict of interest disclosure: Karen Charlton previously received an honorarium to serve on a Malnutrition Advisory Board for Nestle, Australia. No other authors declare a conflict of interest.

\section{References}

1. Russell CA, Elia M. Malnutrition in the UK: where does it begin? Proc Nutr Soc. 2010;69:465-9.

2. BAPEN Malnutrition Advisory Group. The Executive Summary of The 'MUST' report; 2003. (Also available from: http://www.bapen.org.uk/ must_report.html accessed 12/07/2011).

3. Skates JJ, Anthony PS. Identifying geriatric malnutrition in nursing practice: The mini nutritional assessment (MNA®)-An evidence-based screening tool. J Gerontol Nurs. 2012;38:18-27.

4. National Institute for Health and Clinical Excellence. Nutrition support in adults Oral nutrition support, enteral tube feeding and parenteral nutrition; 2006. (Also available from: http://www.nice.org.uk/guidance/cg32/ resources/guidance-nutrition-support-in-adults-pdf accessed 9 February 2011).

5. Schilp J, Kruizenga HM, Wijnhoven HAH, et al. High prevalence of undernutrition in Dutch community-dwelling older individuals. Nutrition. 2012;28:1151-6.

6. Australian and New Zealand Society for Geriatric Medicine. Australian and New Zealand Society for Geriatric Medicine Position Statement No. 6 Under-nutrition and the Older Person. Australas J Ageing. 2009;28:99-105.

7. Watterson C, Fraser A, Banks M, et al. Evidence based practice guidelines for the nutritional management of malnutrition in adult patients across the continuum of care. Nutrition \& Dietetics. 2009;66:S1-34.

8. Kondrup J, Allison SP, Elia M, Vellas B, Plauth M. ESPEN guidelines for nutrition screening 2002. Clin Nutr. 2003;22:415-21.

9. Elia M, Russell CA. Combating malnutrition: recommendations for action: A report from the Advisory Group on Malnutrition, led by BAPEN; 2009. (Also available from: http://www.bapen.org.uk/pdfs/reports/advisory_group_ report.pdf accessed 29 June 2011).

10. BAPEN Malnutrition Advisory Group. The 'MUST' Explanatory Booklet. A Guide to the 'Malnutrition Universal Screening Tool' ('MUST') for Adults; 2003. (Also available from: http:// www.bapen.org.uk/pdfs/must/must explan.pdf accessed 12/07/2011).

11. Hamirudin $\mathrm{AH}, \mathrm{Charlton} \mathrm{K}$, Walton $\mathrm{K}$, et al. 'We are all time poor' Is routine nutrition screening of older patients feasible? Aust Fam Physician. 2013;42:321-6.

12. Flanagan D, Fisher T, Murray M, et al. Managing undernutrition in the elderly: Prevention is better than cure. Aust Fam Physician. 2012;41:695-9.

13. Visvanathan R. Undernutrition and housebound older people. Nutrition and Dietetics. 2009;66:238-42.

14. Hamirudin AH, Charlton K, Walton K, et al. Feasibility of implementing 
routine nutritional screening for older adults in Australian General Practices: A mixed-methods study. BMC Family Practice. 2014;15:186.

15. Nestle Nutrition Institute. Recommendations for Intervention; 2014. (Also available from: http:/ / www.mna-elderly.com/interventions.html accessed $14 / 3 / 2014)$.

16. NSW Health. Eating Well: A Food and Nutrition Resource for Frail Older People and their Carers: NSW Health Central Coast Local Health District; 2011.

17. Kaiser M, Bauer J, Ramsch C, et al. Validation of the Mini Nutritional Assessment short-form (MNA®-SF): A practical tool for identification of nutritional status. J Nutr Health Aging. 2009;13:782-8.

18. Tong A, Sainsbury P, Craig J. Consolidated criteria for reporting qualitative research (COREQ): a 32-item checklist for interviews and focus groups. Int J Qual Health Care. 2007;19:349-57.

19. Wisdom JP, Cavaleri MA, Onwuegbuzie AJ, Green CA. Methodological reporting in qualitative, quantitative, and mixed methods health services research articles. Health Serv Res. 2012;47:721-45.

20. Söderhamn U, Christensson L, Idvall E, Johansson A, Bachrach-Lindström M. Factors associated with nutritional risk in 75-year-old community living people. Int J Older People Nurs. 2012;7:3-10.

21. Visvanathan R, Macintosh C, Callary M, Penhall R, Horowitz M, Chapman I. The nutritional status of 250 older Australian recipients of domiciliary care services and its association with outcomes at 12 months. J Am Geriatr Soc. 2003;51:1007-11.

22. Charlton K, Nichols C, Bowden S, et al. Poor nutritional status of older subacute patients predicts clinical outcomes and mortality at 18 months of follow-up. Eur J Clin Nutr. 2012;66:1224-8.

23. Pomeroy SEM, Cant RP. General practitioners decision to refer patients to dietitians: Insight into the clinical reasoning process. Aust J Prim Health. 2010;16:147-53.
24. Medicare Australia. Quick reference guide for general practitioners; 2014 (Also available from: https: / / www.medicareaustralia.gov.au/provider / business/education/files/2249-1203.pdf accessed 17/10/2014).

25. Leggo M, Banks M, Isenring E, Stewart L, Tweeddale M. A quality improvement nutrition screening and intervention program available to Home and Community Care eligible clients. Nutrition \& Dietetics. 2008;65:162-7.

26. Burge K, Gazibarich B. Nutritional risk among a sample of community-living elderly attending senior citizens' centres. Australian Journal of Nutrition \& Dietetics. 1999;56:137-43.

27. Bauer JM, Kaiser MJ, Sieber CC. Evaluation of nutritional status in older persons: nutritional screening and assessment. Curr Opin Clin Nutr Metab Care. 2010;13:8-13

28. Ülger Z, Halil M, Kalan I, et al. Comprehensive assessment of malnutrition risk and related factors in a large group of community-dwelling older adults. Clin Nutr. 2010;29:507-11.

29. Kaiser MJ, Bauer JM, Rämsch C, et al. Frequency of Malnutrition in Older Adults: A Multinational Perspective Using the Mini Nutritional Assessment. J Am Geriatr Soc. 2010;58:1734-8.

30. Tomstad ST, Söderhamn U, Espnes GA, Söderhamn O. Living alone, receiving help, helplessness, and inactivity are strongly related to risk of undernutrition among older home-dwelling people. International Journal of General Medicine. 2012;5:231-40.

31. Izawa S, Enoki H, Hasegawa J, Hirose T, Kuzuya M. Factors associated with deterioration of mini nutritional assessment-short form status of nursing home residents during a 2-year period. J Nutr Health Aging. 2014;18:372-7.

32. Rist G, Miles G, Karimi L. The presence of malnutrition in communityliving older adults receiving home nursing services. Nutrition and Dietetics. 2012;69:46-50. 